\title{
UN MODELO PARA CURSOS MEGA EN LÍNEA. DESARROLLO, IMPLEMENTACIÓN Y EVALUACIÓN DE UN CURSO EFECTIVO DE APRENDIZAJE EN LÍNEA A GRAN ESCALA
}

\author{
Gunnar Wettergren ${ }^{1}$, Henrik Hansson ${ }^{2}$ \\ Love Ekenberg ${ }^{3}$
}

RESUMEN

La necesidad de una educación superior a nivel global y local se incrementa cada vez más debido a factores sociales, políticos y económicos. Las universidades se enfrentan al desafío de educar grupos crecientes de estudiantes con los mismos o inclusive menos recursos. El uso eficiente de la Tecnología de Información y Comunicación (ICT, por sus siglas en Inglés) y de diseños pedagógicos adaptados, son medios para cumplir este reto. Este documento presenta una ICT a gran escala basada en cursos a distancia en administración de proyectos dictados por el Departamento de Informática y Ciencias de Sistemas, en la Universidad de Estocolmo, Suecia. El curso ha sido dictado siete veces durante el período 2006-2008, con un total de 2.427 estudiantes. La meta de este documento es explorar los siguientes problemas específicos relacionados con cursos mega, en línea: (1) Alta tasa de deserción, (2) Nueva situación de trabajo pedagógico y psicosocial para los profesores, (3) Comunicación y cursos apropiados para este tipo de formato. Además, el documento da un número de sugerencias basadas en nuestras experiencias y conocimientos obtenidos sobre el curso mega en línea. Esto se hace a través del uso de datos recopilados por medio de la plataforma educativa en diferentes bases de datos, entrevistas con profesores involucrados y encuestas realizadas por estudiantes. Algunas de las conclusiones que se presentan en este documento son: los cambios más significativos que influyeron en la tasa de retensión de los cursos fueron el rediseño de la asignación, secuencia clara de eventos a través de las tareas, cursos de autoaprendizaje puestos en práctica que le permiten a los estudiantes aprender las herramientas necesarias para completar las tareas. La pérdida más grande de estudiantes sucedio entre la inscripción en el curso y su comienzo real y cuando el curso pasó del estudio teórico a la aplicación práctica. Errores de diseño menores llevaron a una gran cantidad de trabajo adicional. La necesidad de desarrollar una interfaz de administración efectiva es crucial y esto no debe olvidarse. La situación de trabajo psicosocial para los profesores que manejan los cursos de escala mega en línea se altera significativamente comparada con la enseñanza en aulas.

Palabras clave: ICT, Educación a Distancia, alumnado a gran escala, lecciones aprendidas, diseño.

\footnotetext{
Departamento de Informática y Ciencias de Sistemas, Universidad de Estocolmo /KTH, Foro 100, 16440 Kista, Suecia:

1 E-mail: gunnarw@dsv.su.se

2 E-mail: Henrik.Hansson@dsv.su.se

3 E-mail: lovek@dsv.su.se.
} 


\begin{abstract}
The need for higher education on a global and local level is ever increasing due to social, political and economical factors. Universities are faced with the challenge of educating larger student bodies with the same or even reduced resources. Efficient use of ICT and adapted pedagogical design are means do meet this challenge. This paper presents a large scale Іст based distance course in project management given at the Department of Computer and Systems Sciences, Stockholm University, Sweden. The course has been given seven times during the period 2006-2008, with a total of 2427 students. The aim of this paper is to explore the following specific problems related to online mega courses: (1) The low student completion rate, (2) The changed pedagogical and psycho-social work situation for teachers, (3) Communication and courses suitable for this type of format, furthermore the paper gives a number of suggestions based on our experiences and obtained knowledge about mega online course. This is done through the use of data collected through the educational platform in various databases, interviews with involved teachers, and surveys filled out by students. Some of the conclusions that are presented in this paper are: Most significant changes that influenced completion rate was assignment redesign, clear sequence of events through the assignments, introduced self study courses that enables students to learn the tools needed to complete the assignments. The biggest loss of students was between registering for the course and actually starting the course and when the course moved from theoretical study to practical application. Minor design errors led to huge amount of extra work. The need for development of an effective administrator interface is crucial and must not be forgotten. The psycho-social work situation for online teachers managing mega scale courses are significantly altered as compared to class-room teaching.
\end{abstract}

Key words: ICT, Distance Education, Large scale student body, lessons learned, design.

Recibido: 2 de diciembre de 2008

Aceptado: 6 de abril de 2010

\title{
INTRODUCCIÓN Y OBJETIVO
}

Los cursos universitarios de gran escala en línea se han vuelto mucho más importantes para satisfacer la demanda de educación superior. Las instituciones a nivel mundial están pensando en la Internet, como una solución para sus necesidades educativas. Las regiones en desarrollo lo ven como una forma de educar a la población a un nivel básico y los países desarrollados como un nuevo canal para llegar a estudiantes potenciales.

E-learning (Aprendizaje Virtual) se ha utilizado por mucho tiempo en todo, desde programas de TV educativos que todos vimos cuando estábamos creciendo hasta cursos basados en multimedia utilizando tecnología de punta, y todo lo que está en medio. La educación basada en Internet es el enfoque principal para los cursos mega en los países desarrollados, sin embargo, los cursos por TV, radio y correspondencia se utilizan en muchos países en desarrollo (Durgunheaoğlu,\& Kuşcul, 2008). 
Suecia y Noruega tienen una larga tradición de educación a distancia (Hansson \& Holmberg, 2006, Hansson \& Holmberg 2003). Las primeras escuelas de correspondencia ya habían comenzado a finales del Siglo xIx. Los dos países son grandes pero despoblados y la causa principal para la educación a distancia fue proveer educación a todas las regiones del país. Australia y Canadá son otros dos países con una población diseminada y necesitan difundir la educación a través de distancias muy grandes. Estos países habían desarrollado el aprendizaje asincrónico utilizando comunicación en carta y material impreso mucho antes de los modelos pedagógicos desarrollados para Internet.

El concepto e-learning inicialmente se utilizó en Escandinavia bajo un enfoque pequeño con miras para proveer educación por medio del avance en el estudio individual lineal utilizando a menudo CD-ROM. Sin embargo, en la actualidad los conceptos de e-learning, educación a distancia y educación flexible se fusionaron y su uso se intercambia para educación basada en Internet con tareas colectivas y un modelo pedagógico de constructivismo. El e-learning tradicional se utiliza principalmente en el área de los negocios y de las organizaciones, pero los nuevos enfoques de e-learning utilizando herramientas de la Internet 2 han sido adoptados cada vez más por las Universidades mirando hacia e-learning como una alternativa de cursos regulares en aulas.

En Suecia el número de estudiantes matriculados en educación a distancia se ha triplicado desde 1996/1997. Durante 2005/2006 más de 82.000 estudiantes estaban estudiando cursos a distancia, lo que es equivalente a uno de cada cinco (Agencia Nacional Sueca para Educación Superior/Estadística, Suecia, 2007). El avance hacia e-learning en Suecia está motivado por muchos factores diferentes tales como la globalización, las necesidades financieras, la oportunidad técnica, y el hecho que los profesores quieren ampliar el enfoque pedagógico de la enseñanza (Hansson \& Holmberg, 2003).

El Departamento de Informática y Ciencias de Sistemas (DSV, por sus siglas en inglés) inició en el 2006 (Verano) un curso de administración de Proyectos en línea, denominado Administración de Proyectos Prácticos. La razón principal por la que se inició este curso fue que vimos la gran necesidad, entre los estudiantes, de técnicas en administración de proyectos y fue también en muchos aspectos una prueba para evaluar cómo se pueden administrar y ejecutar los cursos a gran escala (más de 1.000 estudiantes).

A través del uso de cursos mega en línea tal como el descrito en este documento, la educación superior podría abarcar un grupo más amplio de personas que nunca hubieran tenido esta oportunidad de otra manera. También juega un papel importante en la reeducación o para incrementar la educación de personas en el sector profesional debido al hecho que pueden planear y estudiar a su propio ritmo, haciendo que este tipo de educación sea independiente respecto al tiempo y en la ubicación. (Gell \& Cochrane, 1996; Bentley, 1998; Laurillard, 1993; McConnell, 1998).

Los cursos de e-Learning han estado sujetos, por mucho tiempo, al hecho de que hay una tasa más alta de deserción que en los cursos de educación tradicional (Seifert et al., 2008; Arneberg 
et al., 2007). Con la introducción del e-learning el papel del profesor cambió de conferencista clásico a desarrollador, investigador y colaborador (Barajas, Scheuermann y Kikis-Papadakis, 2003). Sin embargo, la mayoría de los estudios se enfocan principalmente en cursos "pequeños". Otro tema importante en la educación en línea es el uso de comunicación asincrónica que por definición no es clara sino ambigua (An \& Frick, 2006).

El objetivo de este documento es explorar los siguientes problemas específicos relacionados con cursos mega en línea:

(1) Alta tasa de deserción de los estudiantes.

(2) Nueva situación pedagógica y psicosocial para los profesores.

(3) Comunicación y cursos apropiados para este tipo de formato.

\section{Estudios previos}

Los estudios de tendencia Mega reportados por Arneberg et al., (2007) y Paulsen (2007) muestran factores cruciales para convertirse en un proveedor exitoso de cursos mega. Inicialmente ellos postularon los siguientes factores como importantes: 1) Factores históricos ${ }^{4}$ 2) Factores tecnológicos ${ }^{5}$ 3) Factores del curso ${ }^{6}$ 4) Factores de administración y de estrategia ${ }^{7}$ 5) Factores económicos. ${ }^{8}$ Además de una buena comercialización basada en un nombre de marca bien conocido y en entusiastas que trabajaron para promover la enseñanza en línea en una fase previa, como base del éxito.

Enoch y Soker (2006) concluyeron que la edad, el género y el origen étnico juegan un papel importante para e-learning.

Berge \& Muilenburg (2005) encontraron ocho factores con barreras estudiantiles hacia el aprendizaje en línea: 1) problemas administrativos, 2) interacción social, 3) capacidades académicas, 4) capacidades técnicas, 5) motivación del alumno, 6) tiempo y apoyo para los estudios, 7) costo y acceso a la Internet, y 8) programas técnicos. Berge et al., realizaron varios estudios previos sobre resistencia del estudiante a la Educación a Distancia (Berge \& Muilenburg, 2003; Berge, Muilenburg \& Haneghan, 2002; Cho \& Berge 2002; Berge \&

4 Larga trayectoria en educación a distancia/en línea/flexible; alta capacidad en educación en línea; desarrollo evolutivo; investigación continua.

5 Alta capacidad en Iст; basado en tecnologías estándar; sistemas ICт bien integrados; sistemas administrativos efectivos.

6 Amplia variedad de materias y niveles; escogencia acertada de temas; inicio y progreso flexible del estudiante; enfoque en comunicación asincrónica

7 Apoyo de los directivos; empleados entusiastas; estrategias que apoyan la educación en línea; enfoque en la calidad; rutinas administrativas efectivas; carga de trabajo predecible y manejable para el profesor; colaboración con otras instituciones; alta credibilidad del gobierno; alguna forma de industrialización.

8 Costo-efectivo; fuentes estables y predecibles de ingresos de la operación; presión sobre la necesidad de cambiar; contratos con tutores y desarrolladores de cursos de medio tiempo. 
Muilenburg, 2001; Muilenburg \& Berge, 2001; Berge \& Muilenburg, 2000; Berge \& Mrozowski,1999; Berge, 1998).

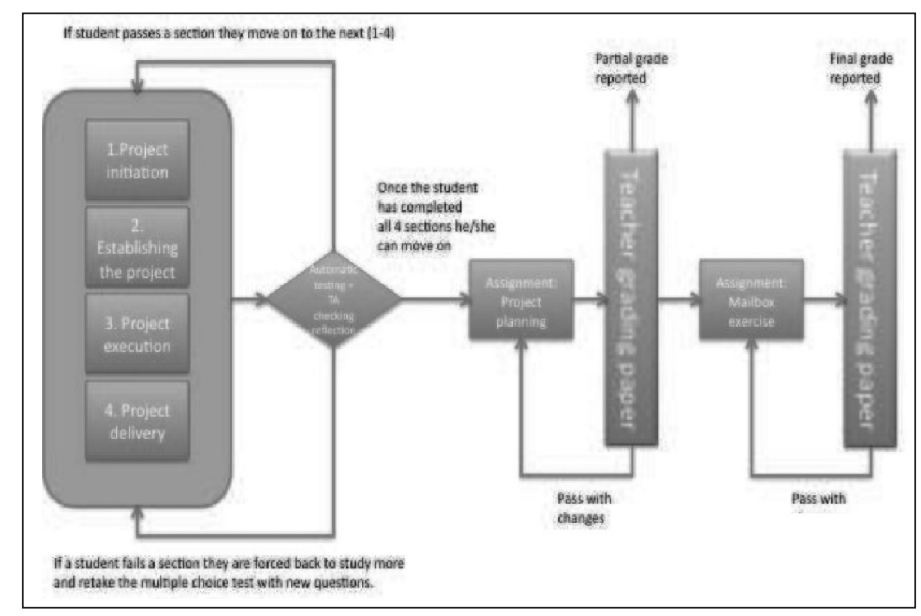

Figura 1. Proceso del Curso

\section{Vistazo general del curso}

El curso se basa exclusivamente en autoaprendizaje y la única interacción entre los estudiantes y los profesores es a través de foros de discusión y mensajes por e-mail. Una vez que el estudiante es aceptado en el curso tiene ocho meses para completarlo. No hay fechas establecidas para la terminación de las tareas sino que se califican y se evalúan de manera ad hoc. Los estudiantes son responsables de manejar su propio tiempo y planear su trabajo tal como lo crean conveniente. Todo el material requerido está disponible en la plataforma educativa y en formato de archivos mp3, por lo tanto, los libros no son necesarios.

El modelo utilizado en este curso es una mezcla de estudios teóricos al leer los materiales o al escuchar grabaciones sonoras de dicho material y ejercicios prácticos en administración de proyectos.

Cuando se diseñó este curso pensamos que era importante no sólo darle a los estudiantes las herramientas necesarias (teoría de lectura) para entender y ser un gerente de proyectos sino también capacitarlos en esas técnicas. Este curso les solicitó a los estudiantes reflexionar e interactuar con otros estudiantes en la discusión de temas sobre administración de proyectos.

A través del curso se les solicitó a los estudiantes realizar un número de tareas diferentes, todas diseñadas para educarlos o para permitirles la aplicación práctica del conocimiento ganado. Las tareas van desde de lectura de textos en el sistema hasta el manejo de trabajo diario de un gerente de proyecto, estas tareas se ilustran en la figura 1 arriba. 
Como este curso contiene dos tipos de tareas diferentes, una teórica y una práctica, necesitamos dos formas separadas de calificar y de examinar a los estudiantes. La parte teórica contiene teoría y reflexiones. Las reflexiones las examina y califica un asistente del profesor que también participa activamente en las discusiones y trata de desafiar a los estudiantes para ver otros aspectos de la discusión. La parte teórica se examina con el uso de preguntas de opción múltiple. Este proceso es $100 \%$ automatizado y el sistema selecciona las preguntas aleatoriamente para cada sección que se entrega al estudiante y las califica cuando los estudiantes oprimen enviar.

La segunda parte del curso, la aplicación teórica se hace a través de dos tareas que se califican como se calificaría cualquier tarea en un curso clásico.

\section{Métodos y datos}

De la gran cantidad de información que alberga cualquier sistema basado en el computador hay que tener en cuenta asimismo la cantidad de material que se puede recopilar sobre los estudiantes. A lo largo de estos dos años se ha acumulado un gran número de datos que resulta imposible presentar en este documento. Los criterios utilizados para la selección de datos fue la siguiente: tenían que ser relevantes respecto a los tres problemas específicos planteados en este documento para analizar y que hubiera suficiente información estadística disponible para obtener respuestas confiables. Los datos se recopilaron de archivos escritos y de diferentes bases de datos que el sistema utiliza para seguirle la pista al progreso y de los resultados del estudiante.

Al comienzo del proceso de escribir este documento establecimos que por su calidad argumentativa, no había necesidad de un análisis estadístico profundo, en cambio utilizamos métodos simples tal como cálculo de frecuencias y datos agregados para comparar los grupos de estudiantes a través del tiempo, la edad, y el género e interpretar esto en relación con la literatura actual y las estadísticas comparables.

Las encuestas utilizadas para obtener las opiniones de los estudiantes fueron una mezcla entre estudiantes que se les solicitó calificar ciertos aspectos en una escala ordinal y preguntas abiertas donde pudieran escribir libremente. Decidimos que esto era el mejor enfoque ya que conocíamos un número de temas que queríamos registrar y que las opiniones de los estudiantes se recopilaban mejor si las escribían en sus propias palabras. La encuesta de respuestas se analizó al calcular distribuciones (preguntas ordinales) y comparar las respuestas de las preguntas abiertas inductivamente para encontrar temas y opiniones comunes que se pudieran generalizar.

La última fuente que utilizamos fue entrevistas con diferentes profesores que han trabajado en el curso a lo largo de los últimos dos años. Estas entrevistas se realizaron en un escenario informal como una discusión donde los encuestados podrían elaborar libremente y dar opiniones sobre diferentes temas respecto al curso y el e-learning en general. Cuando analizamos estos datos de nuevo utilizamos un enfoque inductivo para generalizar con base 
en los temas comunes.

\section{RESULTADOS Y DISCUSIÓN}

En total se matricularon 2.427 en el curso. La distribución de género fue 57.7\% (1.400 estudiantes) mujeres y $42.3 \%$ hombres (1.027 estudiantes). El modo de estudio y el tema atrajo tanto a mujeres como a hombres en números razonablemente equilibrados. Con la excepción del verano de 2007 que atrajo el doble de mujeres (ver figura 2).

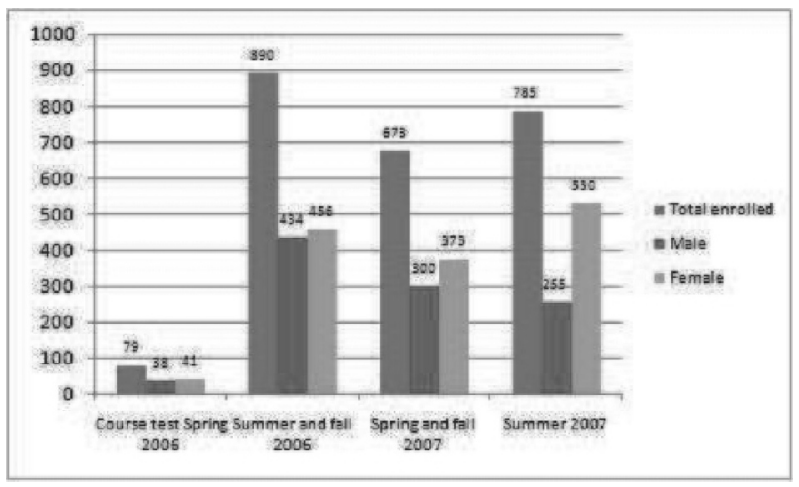

Figura 2. Matrícula sobre ofertas de cursos

\section{Alta tasa de deserción de los estudiantes}

Al examinar los resultados, encontramos que los estudiantes que no terminaban se convertían en un problema de gran magnitud para este tipo de curso. En promedio sobre todas las ofertas del curso, el $39.3 \%$ de los estudiantes matriculados nunca iniciaron o terminaron ninguna tarea. Durante las sesiones que no fueron de verano $(7.1 \%)$ el número parece disminuir comparado con las dos sesiones de verano analizadas (promedio: 55.4\%).

Tabla 1. Matrículas y estudiantes que no iniciaron

\begin{tabular}{|c|c|c|c|c|}
\hline 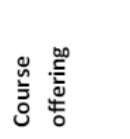 & 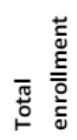 & 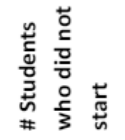 & $\frac{\frac{0}{10}}{\sum}$ & $\begin{array}{l}\frac{\mathscr{\nu}}{\widetilde{N}} \\
\text { हूँ }\end{array}$ \\
\hline $\begin{array}{l}\text { Summer } \\
\text { and Fall } \\
2006\end{array}$ & 890 & $\begin{array}{l}530 \\
(59.5 \%)\end{array}$ & $\begin{array}{l}271 \\
(434) \\
62.4 \%\end{array}$ & $\begin{array}{l}259 \\
(456) \\
56.7 \%\end{array}$ \\
\hline $\begin{array}{l}\text { Spring and } \\
\text { Fall } 2007\end{array}$ & 673 & $48(7.1 \%)$ & $\begin{array}{l}19(300) \\
6.3 \%\end{array}$ & $\begin{array}{l}29(373) \\
7.7 \%\end{array}$ \\
\hline $\begin{array}{l}\text { Summer } \\
2007\end{array}$ & 785 & $\begin{array}{l}403 \\
(51.3 \%)\end{array}$ & $\begin{array}{l}135 \\
(255) \\
52.9 \%\end{array}$ & $\begin{array}{l}268 \\
(530) \\
50.5 \%\end{array}$ \\
\hline Averages & 783 & $39.3 \%$ & $40.5 \%$ & $38.3 \%$ \\
\hline
\end{tabular}


En nuestro caso, la pérdida más grande de estudiantes ocurre antes de que el curso inclusive comience o al inicio, cuando los estudiantes se dan cuenta en qué curso se registraron o cambiaron de parecer. Una explicación de este hecho es que la educación en Suecia es gratis, no se requiere que estudiantes nacionales y extranjeros paguen una matrícula. La financiación de la educación superior en este país, se basa en los impuestos. Por lo tanto, si se tienen las calificaciones necesarias puede llenar una solicitud para cualquier curso y que lo acepten sin tener que pagar. Cuando se trata de educación a distancia el problema se multiplica, es fácil matricularse, hay un gran número de estudiantes, hay poco contacto cara a cara con los profesores, y es difícil que un estudiante obtenga una buena comprensión de los lineamientos de este curso de texto electrónico antes de matricularse. Este es un problema que no tienen que afrontar la mayoría de los países donde la educación no es gratis ya que los estudiantes que pagan por un curso posiblemente terminarán el curso.

Una vez que el curso se ha iniciado, la siguiente pérdida de estudiantes ocurre después de terminar la primera parte. La Parte 1 es una sección teórica donde los estudiantes leen el material en línea, etc. De los estudiantes que terminan la parte 1, un promedio del $54.9 \%$, continúan y completan la parte 2 del curso. Aproximadamente el $90 \%$ de los estudiantes que terminan la parte 2 también terminan la parte 3. La razón principal de esto es, en nuestra opinión, que el paso de leer al respecto y realmente hacerlo es grande y podría inclusive ser un gran reto para muchos estudiantes. Segundo, ya que respondemos a una gran cantidad de estudiantes mayores que trabajan en la industria como profesionales el $43.2 \%$ de las personas que respondieron la encuesta que entregamos después de la Parte 1 dijeron que trabajan y muchos de estos pueden estar interesados en el conocimiento teórico y lo aplicarán a sus diferentes lugares de trabajo.

Al examinar el rendimiento del curso encontramos que los números esperados son mucho más bajos de los que le gustaría a uno ver en un curso de clases regular. En DSV la meta es tener un rendimiento del $70 \%$ (para un curso regular). Cuando examinamos los datos de este curso encontramos que el rendimiento promedio incluyendo a todos los estudiantes fue del $16.6 \%$, el cual es un número muy bajo inclusive para un curso de e-learning. Sin embargo, si tomamos en cuenta que el 39.3\% (promedio) de los que se matriculan nunca inician el curso entonces el rendimiento es del $31.5 \%$. Ver tabla 2 a continuación. Los datos para 2008 fueron incluidos ya que los efectos de muchos de los cambios implementados ocurrieron en el 2008.

Tabla 2. Rendimiento para todas las ofertas del curso

\begin{tabular}{|l|c|c|}
\hline \multicolumn{1}{|c|}{ Oferta del Curso } & Rendimiento & \\
\hline Verano y Otoño 2006 & $16.5 \%$ & $40.8 \%$ \\
\hline Primavera y Otoño 2007 & $14.9 \%$ & $16 \%$ \\
\hline Verano 2007 & $18.5 \%$ & $37.6 \%$ \\
\hline Promedios & $16.6 \%$ & $31.5 \% 1$ \\
\hline 2008 hasta ahora & $23 \%$ & No disponible \\
\hline
\end{tabular}




\begin{tabular}{|l|c|c|}
\hline \multicolumn{1}{|c|}{ Oferta de Curso } & Rendimiento & $\begin{array}{c}\text { Rendimiento sin estudiantes } \\
\text { que nunca iniciaron }\end{array}$ \\
\hline Verano y Otoño de 2006 & $16.5 \%$ & $40.8 \%$ \\
\hline Primavera y Otoño de 2007 & $14.9 \%$ & $16 \%$ \\
\hline Verano 2007 & $18.5 \%$ & $37.6 \%$ \\
\hline Promedios & $16.6 \%$ & $31.5 \% 1$ \\
\hline 2008 hasta ahora & $23 \%$ & No disponible \\
\hline
\end{tabular}

\section{Nueva situación laboral, pedagógica y psicosocial para profesores}

Para entender el ambiente de trabajo psicosocial de los profesores, se realizó un estudio donde los involucrados fueron entrevistados y se les solicitó expresar sus pensamientos sobre el curso a apartir de la siguiente reflexión: cómo es ser un profesor en un ambiente en línea y los diferentes problemas que surgen al enseñar a través de este tipo de medio, puede concluir lo siguiente de las entrevistas:

Un sistema de apoyo técnico administrativo es vital para poder encargarse de las masivas cantidades de estudiantes. Esto es de lejos el área más importante fuera de dictar un buen curso con buenos materiales. Es imposible organizar un curso de esta magnitud sin dedicarle mucho trabajo al diseño de una interfaz administrativa que le ayude a los profesores a manejar esta gran cantidad de estudiantes. Comenzamos con una interfaz muy débil que no estaba diseñada para un grupo grande de estudiantes de cursos bastantes normales (40-100 estudiantes.). La interfaz inicial mostraba a todos los estudiantes en el curso con actividades resaltadas que requerian la atención de los profesores o de los TA's.

Esto fue imposible de manejar ya que la página Web duró 5 minutos en cargar y mostró entre 1.000-1.500 estudiantes a la vez. Se abandonó la vieja interfaz por un enfoque diferente. El punto de inicio fue que sólo se exhibirían las cosas que el profesor necesitaba hacer y se implementó un proceso de calificación donde era más claro en el orden en que se requería hacer cosas. También cambiamos la forma como se exhibía la información al profesor. Inicialmente el profesor necesitaba abrir cada correo individual (en el ejercicio del buzón de correo) calificarlo y abrir el siguiente. En cambio, creamos un proceso donde las respuestas salían en una lista con: la pregunta, la respuesta del estudiante y la respuesta correcta. Al hacer esto disminuimos el tiempo de calificación en más del $60 \%$ e hicimos que el proceso en general fuera mucho más eficiente.

La forma como el curso está organizado y la planeación afecta directamente la situación del profesor. Por ejemplo, en una de las tareas, los estudiantes presentaron un número de documentos de diferentes formatos. Excel, Word, archivos de proyectos. Esto llevó a una gran cantidad de trabajo ya que el profesor que calificaba tenía que bajar los documentos, abrirlos en cuatro programas diferentes. Esto no es problema para un curso con 40 estudiantes aunque puede ser tedioso. Si se tienen más de 3.000 estudiantes es imposible. Para remediar este problema se rediseñó la tarea para que el texto que los estudiantes registraban, estuviera 
ubicado en áreas específicas del documento y forzó a los estudiantes a entregar 2 archivos, un documento de texto y un plan del proyecto; por lo tanto, se redujo el trabajo administrativo en más del $50 \%$.

El papel de la enseñanza, hasta cierto grado, se convirtió en administrativo. Ser profesor de un curso en línea de este tipo es muy diferente al de pararse en frente de los estudiantes en un escenario tradicional. En un curso tradicional se tiene la oportunidad de interactuar con estudiantes cara a cara, ver sus reacciones a medida que presenta el tema en cuestión, etc. Esto no es posible en un escenario en línea. La única forma de comunicarse con los estudiantes es a través de discusiones y e-mail. El papel del profesor en un escenario virtual es más que el de un guía, le muestra a los estudiantes la forma a través de los materiales y le responde preguntas para ayudarlos a superar los problemas y los obstáculos que estos encaran a lo largo del camino. La pregunta es cómo podemos crear un escenario que sea estimulante y desarrollar para profesores cursos, previniendo el peligro de que el trabajo se vuelva monótono y se convierta en administrativo de hecho.

Sería mejor si este tipo de curso lo administrara un equipo de profesores para evitar que la gente se canse del mismo y se queme. Estos tipos de cursos requieren profesores que interactúen con estudiantes de una manera asincrónica, nueva. La mayoría de los profesores no están habituados a este ejercicio y hemos visto la tendencia que los profesores pierdan interés después de dictar el curso por un tiempo. Para evitar este mayor esfuerzo se debe establecer que los estudiantes se ayuden entre sí y que no sólo dependan de la asistencia del profesor.

\section{Cursos de comunicación y cursos apropiados para este tipo de formato}

Para responder esta pregunta decidimos preguntarle a los estudiantes y a los profesores involucrados sobre su opinión respecto a futuros cursos que se organizan de esta manera.

Tabla 3. ¿Le recomendaré este curso a otros?

\begin{tabular}{|c|c|}
\hline $\mathbf{S i}$ & No \\
\hline 445 & 12 \\
\hline $97.4 \%$ & $2.6 \%$ \\
\hline
\end{tabular}

Aunque hubo muchos problemas con las descripciones, la calidad del texto, y el consenso general del proceso de trabajo, parece que a los estudiantes les gusta este formato de curso, $97.4 \%$ de los entrevistados respondieron que lo recomendarían a otros. Esto claramente demuestra que aunque muchas cosas se pueden hacer mejor y de manera más intuitiva para los estudiantes, el concepto general de autoaprendizaje hecho de esta manera funciona y que los estudiantes piensan que es beneficioso. Sobre la pregunta si la falta de un profesor físico dificulta la capacidad de aprender y entender el material el $72 \%$ respondió que no o tenía poco efecto sobre dicha habilidad, lo cual también es alentador. 
Tabla 4. ¿La falta de un profesor físico perjudicó su aprendizaje y entendimiento?

\begin{tabular}{|c|c|c|c|c|c|}
\hline \multicolumn{7}{|l|}{ All } & $\mathbf{2}$ & $\mathbf{3}$ & $\mathbf{4}$ & $\mathbf{5}$ & \multirow{2}{*}{ Very much } \\
\hline Not At & 138 & 58 & 47 & 16 & 8 \\
\hline 193 & $30,0 \%$ & $12,6 \%$ & $10,2 \%$ & $3,5 \%$ & $1,7 \%$ \\
\hline $42,0 \%$ & $* 1$ estudiante no respondió esta pregunta \\
\hline
\end{tabular}

Sobre la pregunta si el DSV debería ofrecer otros cursos de manera similar, la respuesta fue contundentemente afirmativa, el $92.1 \%$ de los estudiantes encuestados pensó que sería una buena idea hacerlo.

Tabla 5. ¿Piensa que el DSV debería ofrecer otros cursos de manera similar?

\begin{tabular}{|c|c|}
\hline Si & No \\
\hline 422 & 36 \\
\hline $92.1 \%$ & $7.9 \%$ \\
\hline
\end{tabular}

*3 estudiantes no respondieron esta pregunta

Hemos establecido que a los estudiantes parece gustarles el formato y que la falta de un profesor no perjudica su aprendizaje. La pregunta siguiente es entonces ¿Qué tipo de cursos deberían dictarse de esta manera?

\section{Si usted respondió sí a las preguntas (Piensa usted que DSV debe ofrecer...) ¿qué tipo de} cursos serían apropiados?

(4) Cursos cortos (10-20) semanas que podrían funcionar como educación mientras que se trabaja o se toman otros cursos.

(5) Cursos técnicos de nivel básico como programación y otros temas relacionados con TI.

(6) Cursos en capacidades específicas que los estudiantes requieran para que puedan hacer el trabajo del curso tal como metodología de la investigación, software, y ambientes de programación.

(7) Las diferentes partes teóricas de casi cualquier curso.

Basado en las respuestas suministradas por profesores y estudiantes se pudieron identificar dos tendencias; la primera es que los estudiantes que trabajan ven esto como una gran herramienta para poder seguir estudiando y mejorar su educación en áreas que necesitan para mantenerse competitivos y también una manera maravillosa de enseñar habilidades específicas tal como un MS Project, Photoshop u otras herramientas necesarias. La segunda tendencia fue que tanto estudiantes como profesores vieron que esto era una forma estupenda de enseñar temas 
básicos como parte de un curso tradicional. Un ejemplo se hizo en este curso. Desde su inicio, en el 2006, forzamos a nuestros estudiantes a utilizar MS Project como parte de sus tareas. Al comienzo no le ayudamos a los estudiantes, más bien asumimos que aprenderían por sí mismos. Esto se convirtió rápidamente en un problema ya que tenían dificultades entendiendo el software y el uso del mismo. Para remediar este problema creamos un curso de autoaprendizaje en $M S$ Project disponible, para ellos, en línea. Esto resultó muy exitoso y vimos mejoras importantes y menos reclamos respecto al MS Project.

\section{CONCLUSIONES}

Las conclusiones principales, relacionadas con las tres áreas de problemas mencionadas anteriormente se presentan en esta sección.

Respecto a la baja tasa de finalización de los estudiantes:

- Inicialmente este curso tenía una tasa de terminación del $16.5 \%$ pero después con las modificaciones la tasa de terminación se incrementó al 23\% (2008)

- Los cambios más significativos fueron: instrucciones más claras y rediseño de las tareas. Con esto se logró que a pesar de la dificultad resultara sencilla la secuencia de eventos necesarios para terminar el curso. Además, introdujo secciones de autoaprendizaje que cubrían las capacidades necesarias en MS Project.

- La mayor deserción de estudiantes ocurre entre el registro y el inicio del curso

- Otro punto de deserción es cuando los estudiantes van de las tareas teóricas a las prácticas

La situación de trabajo del cambio pedagógico y psicosocial para profesores

- Un error de diseño menor llevó a una gran cantidad de trabajo adicional.

- La suposición de que los estudiantes compartían una visión contextual y la dependencia de un texto sólo basado en instrucciones, condujo a equivocaciones y problemas.

- La necesidad de desarrollar una interfaz administradora efectiva es crucial y no debe olvidarse.

- La situación del trabajo psicosocial para profesores en línea que manejan cursos de escala mega se alteran significativamente comparado con la enseñanza en aulas.

Comunicación y cursos apropiados para este tipo de formato 
- Lo estudiantes que terminaron el curso tenían una tasa de satisfacción alta y el $97.2 \%$ de ellos se lo recomendaron a otros.

- Ya que todo el material se prepara y está disponible en línea para los estudiantes, se pueden reasignar los recursos universitarios para resolver problemas ad hoc y con el apoyo de los estudiantes, en vez de lecciones repetitivas sobre conceptos, tareas y contenido básico del curso.

- El 92.1\% de los estudiantes encuestados, pensó que este formato se podría utilizar para otros cursos.

- Los aspectos teóricos de los cursos son particularmente apropiados para este tipo de formato.

\section{NUESTRAS RECOMENDACIONES}

Después de muchos años de organizar este curso se han ganado muchas perspectivas y conocimiento práctico sobre cómo se puede desarrollar. Esta sección esbozará las lecciones más importantes aprendidas (sin ningún orden individual).

- La complejidad de las tareas no es el factor principal del éxito del estudiante sino la claridad de las instrucciones.

- Mantenerse en contacto con estudiantes y recordarles sobre el curso y el hecho de que lo pueden todavía terminar tuvo un gran impacto sobre el rendimiento en nuestro curso.

- Las tareas deben ser ajustadas y rediseñadas para mejorar el curso, no cometer el error de pensar que lo va a tener todo correcto desde el comienzo.

- Cuando se diseñe un curso para grupos grandes de estudiantes la mayor preocupación debe ser la administración y que los profesores sean capaces de hacerle frente a esta cantidad.

- Inicialmente enfocarse en los cursos que sean teóricos por naturaleza y crecer desde ahí.

- Intentar y hacer que los estudiantes se enseñen entre sí al crear la discusión como parte del curso.

- Proveerle a los estudiantes un plan de estudios que esboce el trabajo que deben hacer para establecer cronogramas, aunque sea un curso autodidáctico. Vimos mejores resultados y un rendimiento mucho más alto después de introducir dichos planes.

- Una sugerencia que vamos a implementar es el darle a los estudiantes la capacidad de tomar parte del curso antes de matricularse. La idea es disminuir el número de estudiantes que se matriculan pero que nunca inician el curso. 


\section{INVESTIGACIÓN FUTURA}

Hay aún muchos problemas que no se han resuelto y deben ser investigados en futuros documentos. Esta sección esboza algunos de los temas identificados que necesitan mayor exploración.

- Evaluar los efectos de la falta de un profesor físico sobre la capacidad de aprender.

- Estudios adicionales sobre el rendimiento del curso y los factores que lo afectan.

- Los efectos de nuevos medios tales como el video y el sonido sobre la satisfacción del curso y la capacidad de aprendizaje.

\section{REFERENCIAS BIBLIOGRÁFICAS}

AN. Y. \& FRICK T. (2006) Student Perceptions of Asynchronous Computer-Mediated Communication in Face-to-Face Courses. Journal of Computer-Mediated Communication. P. 485-499. International Communication Association.

ARNEBERG, P., GUÁRDIA, L., KEEGAN, D., LÖSSENKO, J., MÁZÁR, I., MICHELS, P.F., et al. (2007). Analysis of European Mega providers of e-learning. Bekkestua, NKI Publishing House

BARAJAS, M. \& SCHEUERMANN, F. \& KIKIS-PAPADAKIS, K. (2003) Is the role of the Teacher as the knowledge authority in danger in an ICT-learning setting. Elearningeuropa. info. Retrieved September 26, 2008, from http://www.elearningeuropa.info/directory / index.php?page $=$ doc\&doc_id $=580 \&$ docl ng $=6$

BENTLEY, T. (1998) Learning beyond the classroom: education for a changing world (London, Routledge).

BERGE, Z.L. \& MUILENBURG, L.Y. (2003). Barriers to distance education: Perceptions of K-12 educators. Proceedings of the Society for Information Technology and Teacher Education International Conference. Albuquerque, New Mexico USA, March 24-29. Issue 1, pp. 256-259.

BERGE, Z.L., MUILENBURG, L.Y., AND HANEGHAN, J.V. (2002). Barriers to distance education and training: Survey results.

BERGE, Z.L. \& MUILENBURG, L.Y. (2001). Obstacles faced at various stages of capability regarding distance education in institutions of higher learning. Tech Trends 46(4): pp. 40-45. 
BERGE, Z.L. AND MUILENBURG L.Y. (2000). Barriers to distance education as perceived by managers and administrators: Results of a survey. In Melanie Clay (Ed.), Distance Learning Administration Annual 2000.

BERGE, Z.L. \& MROZOWSKI, S. (1999) Barriers to Online Teaching in Elementary, Secondary, And Teacher Education. Canadian Journal of Educational Communication, 27(2): 59-72. Berge, Z.L. (1998). Barriers to online teaching in post-secondary institutions. Online Journal of Distance Education Administration. 1(2).

CHO, S.K. \& BERGE, Z.L. (2002). Overcoming Barriers to Distance Training and Education. Education at a Distance [USDLA Journal] (16)1.

DURGUNOĞLU, A. Y., KUŞCUL, H.,Ö. (2008). Providing access to basic literacy education with educational TV. . European Journal of Open, Distance and E-learning (EURODL).

ENOCH. Y \& SOKER. Z. (2006) Age, gender, ethnicity and the digital divide: university students' use of web-based instructions. London, Routhledge.

GELL, M. \& COCHRANE, P. (1996) Learning and education in an information society, in: W. Dutton (Ed.) Information and communication technologies: visions and realities (Oxford, Oxford University Press).

HANSSON, H \& HOLMBERG, C. (2003). "A European and Swedish perspective on ICT policies and strategies in education. National and institutional pathways: crossings, blind alleys and uphill slopes". Journal of Systemics, Cybernetics and Informatics/

HANSSON, H. \& HOLMBERG, C. (2006). Sweden: Distance education. development and competing paradigms. In: W. Zhang (Ed.). Global Perspectives: Philosophy and Practice in Distance Education (Volume Three), Beijing:China Central Radio \& Television University Press.

LAURILLARD, D. (1993) Rethinking university teaching: a framework for the effective use of educational technology. London, Routledge.

MCCONNELL, D. (1998) Developing networked learning professionals: a critical perspective, in: S.Banks, C. Greabner \& D. McConnell (Eds) Networked lifelong learning (Sheffield, University of Sheffield Press).

MUILENBURG, L.Y., \& BERGE, Z.L. (2005). Student Barriers to Online Learning: A Factor Analytic Study Distance Education: An International Journal, 26(1): pp. 29-48.

MUILENBURG, L.Y. AND BERGE, Z.L. (2001). Barriers to distance education: A factoranalytic study. The American Journal of Distance Education. 15(2): 7-22. 
PAULSEN, M, F. (2007). Megatrends in online distance education - Survey and analyses of European megaproviders of e-learning - Experiences and preliminary results. The conference E-learning in Europe: From projects to practice in Copenhagen 23 - 24 April 2007.

THE SWEDISH NATIONAL AGENCY FOR HIGHER EDUCATION/STATISTICS SWEDEN. (2007). Universitet och högskolor. Studenter och examina i grundutbildning 2005/06. Higher education. Students and graduated students in undergraduate education. The Swedish National Agency for Higher Education/Statistics Sweden.

SEIFERT,T. \& SHEPPARD, B. \& VAUGHN, A.M. (2008). Examining the effectiveness of distance education: Result from multi-level modeling. European Distance and E-learning network (EDEN) Annual conference. 